\title{
Annular pancreas in a toddler
}

\author{
Philipose G. Mulugeta • Melissa A. Hilmes
}

Received: 2 June 2010 /Revised: 16 July 2010 / Accepted: 16 August 2010 /Published online: 18 September 2010

(C) Springer-Verlag 2010

A 2-year-old girl presented to the emergency department with severe abdominal pain, bilious emesis, and elevated serum amylase and lipase levels. Abdominal MRI demonstrates an annular pancreas encircling a collapsed second portion of the duodenum (arrow) (Fig. 1). An MRCP demonstrates the duct of the annular pancreas (arrow), main pancreatic duct (arrowhead) and a dilated common bile duct (dotted arrow) (Fig. 2).

Abnormal fusion of the ventral pancreatic bud to the duodenum is believed to cause annular pancreas. Tethering

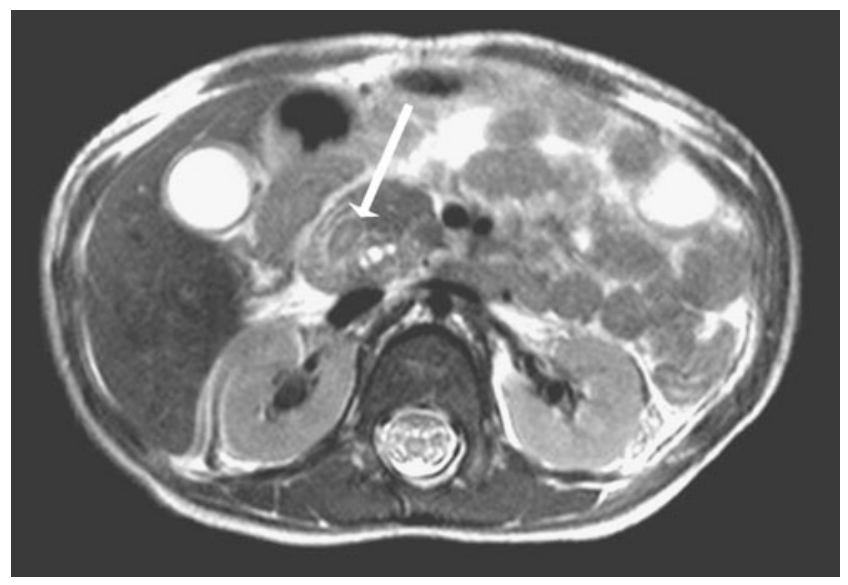

Fig. 1 Axial T2-W MRI through the pancreatic head

P. G. Mulugeta $(\bowtie) \cdot$ M. A. Hilmes

Department of Radiology, Vanderbilt University Medical School,

1211 Medical Center Drive,

Nashville, TN 37232, USA

e-mail: phmulugeta@gmail.com

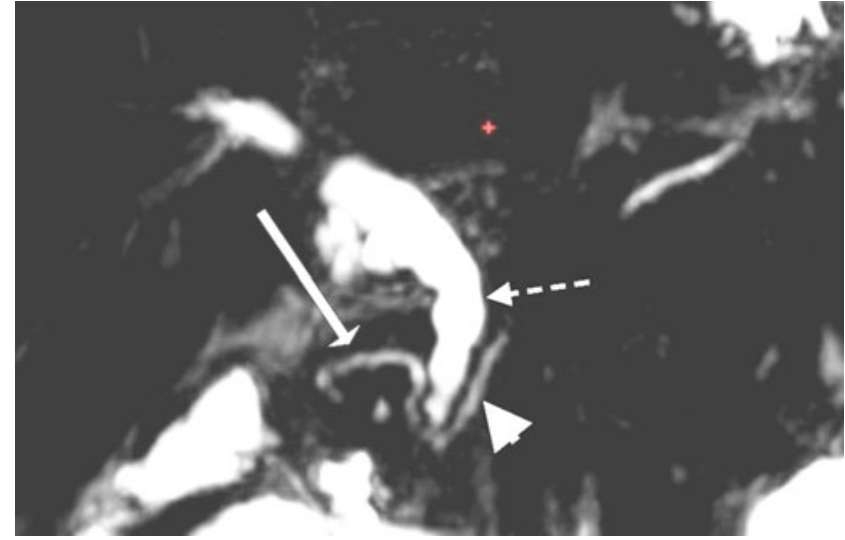

Fig. 2 Coronal MIP reconstruction from MRCP

limits rotation and fusion with the dorsal bud resulting in pancreatic tissue encircling the duodenum. Duodenal obstruction occurs in $10 \%$ of these cases leading to diagnosis in the newborn period [1]. An annular pancreas is also associated with cardiac and intestinal anomalies [2]. A normal upper gastrointestinal series excluded duodenal stenosis in our patient. Her condition was managed with prolonged bowel rest on TPN and she was discharged after tolerating a bland, low-fat diet.

\section{References}

1. Mortele KJ, Rocha TC, Streeter JL et al (2006) Multimodality imaging of pancreatic and biliary congenital anomalies. Radiographics 26:715-732

2. Reinhart RD, Brown JJ, Foglia RP et al (1994) MR-Imaging of annular pancreas. Abdom Imaging 19:301-303 